\title{
Associations of circulating chemerin and adiponectin concentrations with hepatic steatosis
}

\author{
Lena-Maria Levin ${ }^{1}$, Henry Völzke ${ }^{2,3,4}$, Markus M Lerch ${ }^{5}$, Jens-Peter Kühnn ${ }^{6,7}$, Matthias Nauck ${ }^{1,3}$, Nele Friedrich ${ }^{1,3}$ and \\ Stephanie Zylla1,3
}

\author{
${ }^{1}$ Institute of Clinical Chemistry and Laboratory Medicine, University Medicine Greifswald, Greifswald, Germany \\ ${ }^{2}$ Institute for Community Medicine, University Medicine Greifswald, Greifswald, Germany \\ ${ }^{3}$ DZHK (German Centre for Cardiovascular Research), Greifswald, Germany \\ ${ }^{4}$ DZD (German Center for Diabetes Research), Greifswald, Germany \\ ${ }^{5}$ Department of Medicine A, University Medicine Greifswald, Greifswald, Germany \\ ${ }^{6}$ Department of Diagnostic Radiology and Neuroradiology, University Medicine Greifswald, Greifswald, Germany \\ ${ }^{7}$ Institute and Policlinic for Radiology and Interventional Radiology, University Hospital, Carl-Gustav-Carus University Dresden, Dresden, Germany
}

Correspondence should be addressed to S Zylla: stephanie.zylla@med.uni-greifswald.de

\begin{abstract}
Objective: Chemerin and adiponectin are adipokines assumed to be involved in the development of metabolic syndrome-related phenotypes like hepatic steatosis.

We aimed to evaluate the associations of circulating chemerin and adiponectin concentrations with liver enzymes, liver fat content, and hepatic steatosis in the general population.

Methods: Data of 3951 subjects from the population-based Study of Health in Pomerania (SHIP-TREND) were used. Hepatic steatosis was assumed when either a hyperechogenic liver (assessed via ultrasound) or a magnetic resonance imaging (MRI)-quantified liver fat content $>5 \%$ was present. Adjusted sex-specific quantile and logistic regression models were applied to analyze the associations of chemerin and adiponectin with liver enzymes, liver fat content and hepatic steatosis.

Results: The observed associations of chemerin and adiponectin with liver enzymes were very divergent depending on sex, fasting status and the specific enzyme. More consistent results were seen in the analyses of these adipokines in relation to MRI-quantified liver fat content. Here, we observed inverse associations to adiponectin in both sexes as well as a positive (men) or U-shaped (women) association to chemerin. Similarly, the MRIbased definition of hepatic steatosis revealed strongly consistent results: in both sexes, high chemerin concentrations were associated with higher odds of hepatic steatosis, whereas high adiponectin concentrations were associated with lower odds. Conclusion: Our results suggest a role of these adipokines in the pathogenesis of hepatic steatosis independent of metabolic or inflammatory disorders. However, experimental studies are needed to further clarify the underlying mechanisms and the inter-play between adipokine concentrations and hepatic steatosis.
\end{abstract}
Key Words
- adiponectin
- chemerin
- hepatic steatosis
- liver fat content
- liver enzymes

https://ec.bioscientifica.com

https://doi.org/10.1530/EC-19-0300 (c) 2019 The authors Published by Bioscientifica Ltd

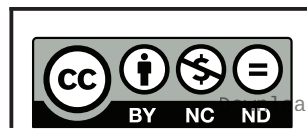

This work is licensed under a Creative Commons Attribution-NonCommercial-NoDerivatives 4.0 Internationab ticense.ifica.com at 04/26/2023 12:56:50PM
Endocrine Connections (2019) 8, 1097-1107 


\section{Introduction}

In industrialized countries, the prevalence and incidence of hepatic steatosis have steadily increased over the past decades (1). Besides excessive alcohol consumption, obesity and other metabolic disorders have been frequently identified as potential risk factors for fat accumulation in the liver (2). Therefore, hepatic steatosis is viewed as the hepatic manifestation of the metabolic syndrome (3).

Previous research in obesity and its metabolic consequences has mainly focused on the role of adipose tissue as an endocrine organ that produces a multitude of metabolically active hormones, so-called adipokines, which are known to be involved in the regulation of glucose and lipid metabolism, food intake and inflammation (4, $5)$. Because adipokines affect insulin sensitivity in the liver (4) and their circulating concentrations are altered in patients with nonalcoholic fatty liver disease (NAFLD) $(3,4)$, they have been considered to play a role in the pathogenesis of hepatic steatosis.

Furthermore, adipokines have been considered to play a role in the pathogenesis of hepatic steatosis, because they affect insulin sensitivity in the liver (4) and their circulating concentrations are altered in patients with NAFLD $(3,4)$.

The adipokine chemerin is encoded by RARRES 2 (retinoic acid receptor responder 2) and exerts its biological effects mainly via the chemokine-like receptor 1 (CMKLR1) (5). In humans, chemerin is known to regulate the cell differentiation from preadipocytes to adipocytes (6). Consequently, many studies have observed positive associations of circulating chemerin concentrations with obesity $(6,7,8)$ and associated metabolic diseases $(6,9)$. Besides their expression in adipose tissue, chemerin and CMKLR1 have been found to be expressed in various other human tissues and cell populations, including the liver (6), Kupffer cells (10) and primary human hepatocytes (10).

Based on these findings, an association between circulating chemerin concentrations and hepatic steatosis is assumed. However, up to now, only few clinical studies have focused on the link between circulating chemerin concentrations and liver function, and these studies have reported conflicting findings $(7,8,11,12,13,14,15)$. Some studies have detected higher circulating chemerin concentrations in NAFLD patients than in healthy controls $(11,13,14)$, whereas others did not confirm this finding $(12,15)$. Likewise, studies dealing with the relation between serum chemerin concentrations and different liver enzymes, such as alanine aminotransferase (ALT), aspartate aminotransferase (AST) or gammaglutamyltransferase (GGT), have observed conflicting results $(8,15)$.

The situation looks rather different when we look at adiponectin, the most abundant adipokine inside the human body (16). Adiponectin is primarily synthesized and released from adipose tissue (16) and is known to exert its biological effects mainly via AdipoR1 and AdipoR2 - two transmembrane receptors (16). AdipoR1 is mostly expressed in the skeletal muscle, whereas AdipoR2 is predominantly found in the liver (16). Interestingly, human adiponectin has been observed to be inversely associated with obesity as well as with the hepatic fat content $(17,18,19)$. Hence, existing studies have detected lower circulating adiponectin concentrations in subjects with hepatic steatosis than in healthy controls $(14,17$, 20). Likewise, inverse correlations between human adiponectin and ALT, AST, alkaline phosphatase (AP) as well as GGT have been reported $(17,18,20,21)$.

Actually, there is a lack of consistent results concerning the association of chemerin with hepatic steatosis. Therefore, the aim of the present study was to use data from a large and well-characterized study population to investigate the potential link of circulating chemerin concentrations with circulating concentrations of liver enzymes, MRI-quantified liver fat content and hepatic steatosis (defined by an ultrasonographically assessed hyperechogenic liver or a liver fat content $>5 \%$ ). To compare and validate these results with another well-studied adipokine, we additionally analyzed circulating adiponectin concentrations in the context of hepatic steatosis.

\section{Materials and methods}

\section{Study population}

The Study of Health in Pomerania (SHIP) is a populationbased study conducted in West Pomerania, a rural region in the northeast of Germany (22). So far, the overall research project consists of two separate cohorts (SHIP and SHIP-TREND). The present study is based on data from SHIP-TREND. In SHIP-TREND, a stratified random sample of 8826 persons, aged 20-79 years, was drawn from the central population registry of the German Federal State of Mecklenburg-West Pomerania. The baseline examinations were conducted between 2008 and 2012. Overall, 4420 of the invited adults chose to participate (50.1\% response). The study followed the principles of the Declaration of 
Helsinki and was approved by the ethics committee of the University of Greifswald. All participants provided written informed consent. SHIP data are publically available for scientific and quality control purposes and data usage can be applied for via www.community-medicine.de.

From the 4420 subjects available for analyses, pregnant women $(n=10)$ and subjects with missing data in chemerin or adiponectin concentrations $(n=146)$ or in any of the confounding or outcome variables $(n=313)$ were excluded from the study. The final study population available for the present analyses consisted of 3951 subjects (51.25\% women) aged 20-84 years. Data on the MRI-based quantification of liver fat content were available in a subsample consisting of 1735 subjects (51.59\% women).

\section{Measurements}

Data on age, sex, sociodemographic characteristics and medical histories were obtained by standardized computer-assisted personal interviews. Smoking status was categorized into current, former or never having smoked. Mean daily alcohol consumption was calculated using beverage-specific pure ethanol volume proportions. Height, weight and waist circumference were quantified following a standardized protocol. The weight and height of each subject were used to calculate its body mass index $\left(\mathrm{kg} / \mathrm{m}^{2}\right)$. After an initial 5-min resting period, blood pressure was measured three times on seating subjects using a digital blood pressure monitor (HEM705CP; Omron, Tokyo, Japan), with each reading being followed by a further resting period of $3 \mathrm{~min}$. The mean of the second and third measurements was used for these analyses.

Blood samples were collected between 7:00 $\mathrm{h}$ and 13:00 h from the cubital vein. With $61.71 \%(n=2438)$, the majority of the observed subjects were fasting at the time of the blood sampling. This means they have not been eating or drinking (except water) for at least $8 \mathrm{~h}$. Aliquots were prepared for immediate analysis and for storage at $-80^{\circ} \mathrm{C}$. Serum ALT, AST, AP, GGT, total triglycerides, serum glucose and high-sensitivity $\mathrm{C}$-reactive protein (hsCRP) were measured using the Dimension Vista 500 analytical system (Siemens AG). Glycated hemoglobin (HbA1c) was determined by high-performance liquid chromatography with spectrophotometric detection (Diamat Analyzer; Bio-Rad). Plasma chemerin and serum adiponectin concentrations were assessed using a commercially available ELISA technique (Mediagnost, Reutlingen, Germany). The detection limits of the assays were $0.005 \mathrm{ng} / \mathrm{mL}$ and $0.0006 \mu \mathrm{g} / \mathrm{mL}$ for chemerin and adiponectin concentrations, respectively. The interassay coefficients of variation for chemerin were 5.8 and $5.5 \%$ and for adiponectin 6.8 and $6.2 \%$ for low and high concentrations, respectively.

Sonographic examinations of the liver were performed by trained physicians with a portable device using a 13-MHz linear array transducer (vivid-I, General Electrics, Frankfurt, Germany). The examiners were not informed about the subject's clinical and laboratory characteristics. A hyperechogenic pattern was defined as the presence of an ultrasonographic 'bright liver' (with evident contrast between hepatic and renal parenchyma) (23).

The quantification of liver fat content was done by MRI examination which was performed on a commercial 1.5-Tesla MR system (Magnetom Avanto, Siemens Healthcare AG). Liver fat content was assessed using a three-echo chemical shift encoded MRI of the liver. Postprocessing of MRI data was performed and the protondensity fat fraction was acquired (24). At this time, the proton-density fat fraction is the non-invasive reference for assessment of liver fat (25). Livers proton-density fat fraction was measured with a region of interest (ROI) placed in the center of the liver. Vessels and artifacts were excluded from the ROI. Based on a previous calibration of proton-density fat fraction and histopathology (26), individuals who had more than 5\% liver fat content were classified as subjects with evidence for hepatic steatosis.

For statistical analyses, two different definitions of hepatic steatosis were used. In the first setting, hepatic steatosis was defined by the presence of an ultrasonographically assessed hyperechogenic liver (US'; $n=1598)$. In the second setting, hepatic steatosis was defined based on MRI examinations: subjects with more than $5 \%$ liver fat content were classified as having hepatic steatosis $(n=726)$.

\section{Statistical analyses}

Continuous data were expressed as median (25th-75th quartile), and nominal data were given as percentage. For bivariate statistics, Mann-Whitney $U$ test (interval data) or chi-square test (nominal data) was used to compare men and women. The associations of circulating chemerin and adiponectin concentrations (as exposure variable) with serum liver enzymes (ALT, AST, AP, GGT) or liver fat content were assessed by multivariable quantile regression models. All analyses were done separately for men and women as initial regression models detected statistically significant interaction effects between chemerin and 
Table 1 Descriptive statistics of the study population.

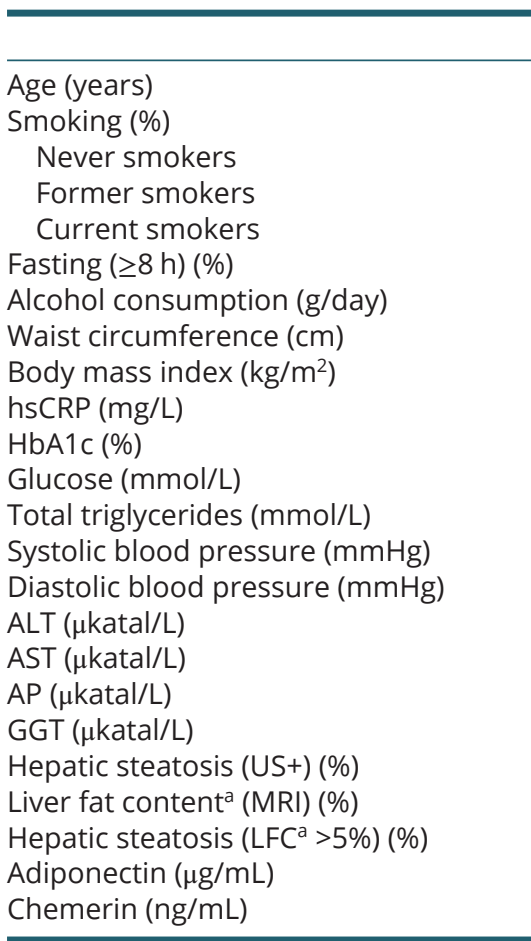

\begin{tabular}{c}
\hline Men $(n=1926)$ \\
$53(41-65)$ \\
24.87 \\
45.79 \\
29.34 \\
58.62 \\
$7.9(2.1-18.5)$ \\
$97(89-106)$ \\
$28(26-31)$ \\
$1.2(0.6-2.5)$ \\
$5.3(5.0-5.8)$ \\
$5.6(5.2-6.2)$ \\
$1.6(1.1-2.3)$ \\
$133(124-144)$ \\
$80(73-86)$ \\
$0.46(0.34-0.64)$ \\
$0.33(0.26-0.41)$ \\
$1.10(0.96-1.30)$ \\
$0.64(0.47-0.94)$ \\
47.09 \\
$4.95(2.84-9.26)$ \\
49.29 \\
$5.4(3.7-7.6)$ \\
$95.4(80.5-113.9)$
\end{tabular}

\begin{tabular}{cccc}
\hline Women $(n=2025)$ & & $\boldsymbol{P}$ \\
$52(40-64)$ & & $<0.01$ \\
& & $<0.01$ \\
29.76 & & \\
24.14 & & \\
64.64 & & $<0.01$ \\
$1.8(0.4-4.9)$ & & $<0.01$ \\
$84(75-94)$ & $<0.01$ \\
$27(24-31)$ & $<0.01$ \\
$1.5(0.8-3.4)$ & $<0.01$ \\
$5.2(4.8-5.6)$ & $<0.01$ \\
$5.2(4.9-5.7)$ & $<0.01$ \\
$1.3(0.9-1.8)$ & $<0.01$ \\
$120(110-133)$ & $<0.01$ \\
$74(69-81)$ & $<0.01$ \\
$0.31(0.24-0.41)$ & $<0.01$ \\
$0.26(0.21-0.33)$ & $<0.01$ \\
$1.10(0.89-1.40)$ & $<0.01$ \\
$0.43(0.35-0.56)$ & $<0.01$ \\
34.12 & $<0.01$ \\
$3.25(2.15-6.93)$ & $<0.01$ \\
34.86 & $<0.01$ \\
$8.9(6.2-12.2)$ & \\
$102.4(85.7-122.0)$ & \\
& & \\
\hline
\end{tabular}

Continuous data are expressed as median (1st-3rd quartile); nominal data are given as percentages. Chi-square test (nominal data) or Mann-Whitney $U$ test (continuous data) was used for comparisons between men and women.

aMagnetic resonance imaging (MRI) examinations were performed in a subsample of 1735 subjects.

ALT, alanine aminotransferase; AP, alkaline phosphatase; AST, aspartate aminotransferase; GGT, gamma-glutamyl-transferase; HbA1c, glycated hemoglobin; hsCRP, high-sensitivity C-reactive protein; LFC, liver fat content; US', presence of an hyperechogenic liver defined via ultrasonography (US).

adiponectin with sex. Possible non-linear associations were tested in all models by including restricted cubic splines with three knots located at the 5th, 50th and 95th percentiles. If the likelihood ratio test indicated a significant increase in model fitness, the spline term for chemerin or adiponectin was included in the regression model. All regression models were adjusted for age, waist circumference, $\mathrm{HbA1c}$, total triglycerides, systolic blood pressure and hsCRP. Likewise, logistic regression models were performed to analyze the associations of circulating chemerin and adiponectin with the two different definitions of hepatic steatosis. To avoid very small coefficients in the regression analyses, we always modeled the change per standard deviation (s.D.) for chemerin (S.D. $=29 \mathrm{ng} / \mathrm{mL}$ ) and adiponectin (s.D. = $5 \mu \mathrm{g} / \mathrm{mL}$ ) concentrations, respectively.

In order to examine any potential influence of the fasting status on the observed associations, all analyses were repeated after exclusion of non-fasting subjects $(n=1513)$. Furthermore, the results were validated in a subpopulation without diabetes. The latter was done, in the light of previous studies where an impact of medications for the treatment of type 2 diabetes (e.g., metformin, rosiglitazone or pioglitazone) on chemerin and adiponectin concentrations has been reported $(19,27)$. Therefore, all subjects taking anti-diabetic drugs (classified according to anatomic, therapeutic and chemical (ATC) code A10) or with HbA1c concentrations $\geq 6.5 \%$ were excluded $(n=408)$. Additionally, we examined the potential influence of alcohol consumption or known liver disease within a further sensitivity analysis by excluding subjects with heavy alcohol consumption (men $>30 \mathrm{~g}$ /day, women $>20 \mathrm{~g} /$ day), a self-reported liver disease (hepatitis, fatty liver, gallstones) or taking bile and liver drugs (ATC: A05; $n=662$ ).

Statistical significance was assumed at a $P$ value $<0.05$ and for interactions at a $P$ value $<0.1$. Statistical analyses were performed using SAS 9.4 (SAS Institute Inc.).

\section{Results}

\section{General characteristics of the study sample}

General characteristics of the study population stratified by sex are presented in Table 1 . Women were more often never smokers and consumed less alcohol than men. 
With respect to metabolic syndrome-related parameters, women showed lower concentrations of total triglycerides, HbA1c, glucose, blood pressure as well as a smaller waist circumference and a lower body mass index compared to men. However, women exhibited higher serum concentrations of hsCRP than men. The prevalence of ultrasound-based defined hepatic steatosis was $47.09 \%$ in men and $34.12 \%$ in women. This higher prevalence for men was also reflected in the MRI-quantified liver fat content and serum concentrations of ALT, AST, AP and GGT. Circulating chemerin and adiponectin concentrations were significantly higher in women than in men (Table 1). No fasting-dependent or diurnal effects on adipokine concentrations were observed (data not shown).

\section{Association of plasma chemerin with liver enzymes, MRI-quantified liver fat content and different definitions of hepatic steatosis}

Quantile regression analyses revealed a positive association between chemerin and the liver enzyme AP in both sexes. The bell-shaped association of chemerin with ALT and GGT was observed only in men. The positive association between chemerin and AST was detected in women, but not in men. When only considering fasting subjects, it can be observed that the significant associations between chemerin and liver enzymes seen before mainly disappeared. Only the positive association between chemerin and AP in women remained (Table 2). After exclusion of subjects taking anti-diabetic drugs or with HbA1c concentrations $\geq 6.5 \%$, the bell-shaped association between chemerin and ALT seen in men changed to a non-significant positive one (data not shown). The bellshaped association of chemerin with GGT observed in men changed to a non-significant positive one after exclusion of subjects with heavy alcohol consumption or reported liver disease (data not shown).

The association between chemerin and MRIquantified liver fat content was strictly positive in men, whereas women showed a U-shaped association (Fig. 1 and Table 2). These associations remained stable in all sensitivity analyses.

Fully adjusted logistic regression models were performed to assess the odds of hepatic steatosis (determined by two different definitions) depending on chemerin concentrations. In men, each increase in plasma chemerin per $29 \mathrm{ng} / \mathrm{mL}$ was associated with $16 \%$ higher odds of ultrasound-based defined hepatic steatosis. In contrast, no significant association between chemerin and ultrasound-based defined hepatic steatosis

Table 2 Results from sex-specific quantile regression analyses: associations of circulating chemerin and adiponectin concentrations with liver enzymes and MRI-quantified liver fat content (LFC).

\begin{tabular}{|c|c|c|c|c|c|c|c|c|c|}
\hline & & \multicolumn{4}{|c|}{ Men $(n=1926)$} & \multicolumn{4}{|c|}{ Fasting men $(n=1129)$} \\
\hline & & \multicolumn{2}{|c|}{ Chemerin per $29 \mathrm{ng} / \mathrm{mL}$} & \multicolumn{2}{|c|}{ Adiponectin per $5 \mu \mathrm{g} / \mathrm{mL}$} & \multicolumn{2}{|c|}{ Chemerin per $29 \mathrm{ng} / \mathrm{mL}$} & \multicolumn{2}{|c|}{ Adiponectin per $5 \mu \mathrm{g} / \mathrm{mL}$} \\
\hline & & Beta (StdErr) & $P$ & Beta (StdErr) & $P$ & Beta (StdErr) & $P$ & Beta (StdErr) & $P$ \\
\hline \multirow[t]{2}{*}{$\mathrm{ALT}$} & $\operatorname{lin}$ & $0.042(0.012)$ & $<0.01$ & $-0.058(0.020)$ & $<0.01$ & $0.012(0.008)$ & 0.17 & $-0.009(0.008)$ & 0.24 \\
\hline & spl & $-0.007(0.002)$ & $<0.01$ & $0.016(0.006)$ & $<0.01$ & - & & & \\
\hline \multirow[t]{2}{*}{ AST } & $\operatorname{lin}$ & $-0.005(0.005)$ & 0.28 & $0.011(0.005)$ & 0.02 & $-0.002(0.006)$ & 0.79 & $-0.025(0.018)$ & 0.16 \\
\hline & spl & - & & - & & - & & $0.017(0.006)$ & $<0.01$ \\
\hline AP & $\operatorname{lin}$ & $0.025(0.007)$ & $<0.01$ & $0.012(0.012)$ & 0.31 & $0.018(0.010)$ & 0.07 & $0.007(0.017)$ & 0.69 \\
\hline \multirow[t]{2}{*}{ GGT } & $\operatorname{lin}$ & $0.054(0.016)$ & $<0.01$ & $0.009(0.011)$ & 0.40 & $0.016(0.013)$ & 0.21 & 0.009 (0.013) & 0.47 \\
\hline & spl & $-0.008(0.002)$ & $<0.01$ & - & & - & & - & \\
\hline \multirow[t]{4}{*}{$\mathrm{LFC}^{\mathrm{a}}$} & Lin & $0.585(0.216)$ & $<0.01$ & $-0.825(0.171)$ & $<0.01$ & $0.672(0.258)$ & $<0.01$ & $-0.827(0.207)$ & $<0.01$ \\
\hline & & \multicolumn{4}{|c|}{ Women $(n=2025)$} & \multicolumn{4}{|c|}{ Fasting women $(n=1309)$} \\
\hline & & \multicolumn{2}{|c|}{ Chemerin per $29 \mathrm{ng} / \mathrm{mL}$} & \multicolumn{2}{|c|}{ Adiponectin per $5 \mu \mathrm{g} / \mathrm{mL}$} & \multicolumn{2}{|c|}{ Chemerin per $29 \mathrm{ng} / \mathrm{mL}$} & \multicolumn{2}{|c|}{ Adiponectin per $5 \mu \mathrm{g} / \mathrm{mL}$} \\
\hline & & Beta (StdErr) & $P$ & Beta (StdErr) & $P$ & Beta (StdErr) & $P$ & Beta (StdErr) & $P$ \\
\hline $\mathrm{ALT}$ & $\operatorname{lin}$ & $0.004(0.004)$ & 0.24 & $-0.003(0.003)$ & 0.26 & 0.009 (0.005) & 0.07 & $-0.003(0.004)$ & 0.38 \\
\hline AST & lin & $0.005(0.003)$ & 0.05 & $0.006(0.002)$ & 0.01 & $0.005(0.004)$ & 0.14 & $0.010(0.003)$ & $<0.01$ \\
\hline AP & lin & $0.038(0.010)$ & $<0.01$ & $0.016(0.008)$ & 0.06 & $0.042(0.012)$ & $<0.01$ & $0.011(0.011)$ & 0.28 \\
\hline GGT & lin & $0.010(0.005)$ & 0.04 & $-0.007(0.003)$ & 0.04 & 0.009 (0.006) & 0.15 & $-0.005(0.004)$ & 0.25 \\
\hline \multirow[t]{2}{*}{$\mathrm{LFC}^{\mathrm{a}}$} & $\operatorname{lin}$ & $-0.554(0.190)$ & $<0.01$ & $-0.516(0.068)$ & $<0.01$ & $-0.879(0.264)$ & $<0.01$ & $-0.324(0.080)$ & $<0.01$ \\
\hline & spl & $0.149(0.045)$ & $<0.01$ & - & & 0.198 (0.058) & $<0.01$ & - & \\
\hline
\end{tabular}

Quantile regression was used to predict the median. The estimated coefficients (beta), standard errors (StdErr) and $P$ values are given. Quantile regression models were adjusted for age, waist circumference, glycated hemoglobin, systolic blood pressure, total triglycerides and high-sensitivity C-reactive protein. A spline term (spl) was added to the linear term (lin) of the respective adipokine when the usage of splines represented a gain in model quality as assessed by a likelihood ratio test.

aMagnetic resonance imaging (MRI) examinations were performed in a subsample of 840 men (577 of them fasting) and 895 women (686 of them fasting). ALT, alanine aminotransferase; AP, alkaline phosphatase; AST, aspartate aminotransferase; GGT, gamma-glutamyl-transferase.

https://ec.bioscientifica.com https://doi.org/10.1530/EC-19-0300 (c) 2019 The authors Published by Bioscientifica Ltd

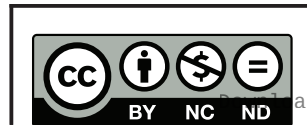

This work is licensed under a Creative Commons Attribution-NonCommercial-NoDerivatives 4.0 Internationab bicense.ifica. com at 04/26/2023 12:56:50PM 

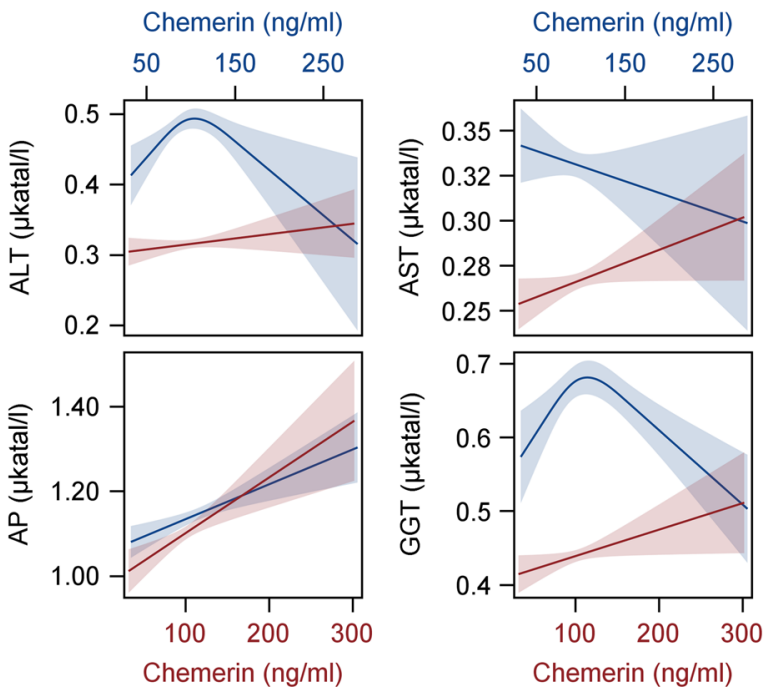

was seen for women (Table 3). When hepatic steatosis was defined by MRI-quantified liver fat content, a significant association with chemerin was seen in both sexes after adjustment for the full risk factor profile (Table 3). The results of fasting subjects were clearly comparable with the results presented for the whole study population (Table 3). Furthermore, the presented effect estimates remained stable when repeating the analyses in the other subpopulations (data not shown).
Figure 1

Associations of plasma chemerin concentrations with liver enzymes and liver fat content (LFC) as calculated by sex-specific quantile regression analyses. Presented is the predicted median together with its corresponding 95\% confidence interval separately for men (blue, $x 2$ axis) and women (red, $x 1$ axis). Quantile regression models were adjusted for age, waist circumference, glycated hemoglobin, systolic blood pressure, total triglycerides and high-sensitivity C-reactive protein. *Magnetic resonance imaging examinations were performed in a subsample of 840 men and 895 women. ALT, alanine aminotransferase; AP, alkaline phosphatase; AST, aspartate aminotransferase; GGT, gamma glutamyl transferase.

\section{Association of serum adiponectin with liver enzymes, MRI-quantified liver fat content and different definitions of hepatic steatosis}

Fully adjusted quantile regression models revealed a positive association between adiponectin and AST in both sexes but no association with AP. A U-shaped association was observed between adiponectin and ALT in men, whereas no associations between these

Table 3 Results from sex-specific logistic regression analyses: associations of circulating chemerin and adiponectin with the odds of hepatic steatosis as defined by two different criteria.

\begin{tabular}{|c|c|c|c|c|c|c|}
\hline & & \multirow[b]{2}{*}{ Cases } & \multicolumn{2}{|c|}{ Chemerin per $29 \mathrm{ng} / \mathrm{mL}$} & \multicolumn{2}{|c|}{ Adiponectin per $5 \mu \mathrm{g} / \mathrm{mL}$} \\
\hline & & & OR $(95 \% \mathrm{Cl})$ & $P$ & OR $(95 \% \mathrm{Cl})$ & $P$ \\
\hline \multicolumn{7}{|c|}{$\overline{\text { Hepatic steatosis defined based on ultrasound (US) }}$} \\
\hline \multirow[t]{2}{*}{ Men } & US $^{-}$ & 1019 & Ref. & & Ref. & \\
\hline & $\mathrm{US}^{+}$ & 907 & $1.16(1.02-1.31)$ & 0.02 & $0.56(0.47-0.66)$ & $<0.01$ \\
\hline \multirow[t]{2}{*}{ Fasting men } & US $^{-}$ & 602 & Ref. & & Ref. & \\
\hline & US $^{+}$ & 527 & $1.24(1.05-1.46)$ & 0.01 & $0.60(0.48-0.74)$ & $<0.01$ \\
\hline \multirow[t]{2}{*}{ Women } & US $^{-}$ & 1334 & Ref. & & Ref. & \\
\hline & US $^{+}$ & 691 & $0.97(0.86-1.10)$ & 0.65 & $0.79(0.70-0.89)$ & $<0.01$ \\
\hline \multirow[t]{2}{*}{ Fasting women } & US $^{-}$ & 871 & Ref. & & Ref. & \\
\hline & US $^{+}$ & 438 & $1.04(0.88-1.23)$ & 0.65 & $0.86(0.74-0.99)$ & 0.05 \\
\hline \multicolumn{7}{|c|}{ Hepatic steatosis defined based on liver fat content (LFC-MRI) } \\
\hline \multirow[t]{2}{*}{ Men } & $\mathrm{LFC}^{\mathrm{a}} \leq 5 \%$ & 426 & Ref. & & Ref. & \\
\hline & $\mathrm{LFC}^{\mathrm{a}}>5 \%$ & 414 & $1.42(1.14-1.76)$ & $<0.01$ & $0.52(0.40-0.69)$ & $<0.01$ \\
\hline \multirow[t]{2}{*}{ Fasting men } & $\mathrm{LFC}^{\mathrm{a}} \leq 5 \%$ & 295 & Ref. & & Ref. & \\
\hline & $\mathrm{LFC}^{\mathrm{a}}>5 \%$ & 282 & $1.34(1.02-1.75)$ & 0.03 & $0.53(0.38-0.75)$ & $<0.01$ \\
\hline \multirow[t]{2}{*}{ Women } & $\mathrm{LFC}^{\mathrm{a}} \leq 5 \%$ & 583 & Ref. & & Ref. & \\
\hline & $\mathrm{LFC}^{\mathrm{a}}>5 \%$ & 312 & $1.36(1.09-1.71)$ & $<0.01$ & $0.58(0.47-0.72)$ & $<0.01$ \\
\hline \multirow[t]{2}{*}{ Fasting women } & $\mathrm{LFC}^{\mathrm{a}} \leq 5 \%$ & 459 & Ref. & & Ref. & \\
\hline & $\mathrm{LFC}^{\mathrm{a}}>5 \%$ & 227 & $1.37(1.05-1.80)$ & 0.02 & $0.65(0.51-0.84)$ & $<0.01$ \\
\hline
\end{tabular}

Presented are the odds ratios (OR), together with the $95 \%$ confidence interval $(95 \% \mathrm{Cl})$ and the $P$ value. Logistic regression models were adjusted for age, waist circumference, glycated hemoglobin, systolic blood pressure, total triglycerides and high-sensitivity C-reactive protein.

a Magnetic resonance imaging (MRI) examinations were performed in a subsample of 840 men (577 of them fasting) and 895 women (686 of them fasting). LFC, liver fat content; ref, reference group; US ${ }^{+}$, presence of a hyperechogenic liver defined via ultrasonography (US); US', no presence of a hyperechogenic liver.

https://ec.bioscientifica.com https://doi.org/10.1530/EC-19-0300 (c) 2019 The authors Published by Bioscientifica Ltd

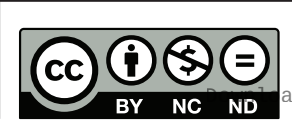

This work is licensed under a Creative Commons Attribution-NonCommercial-NoDerivatives 4.0 elnternationad ticense ifica.com at 04/26/2023 12:56:50PM 

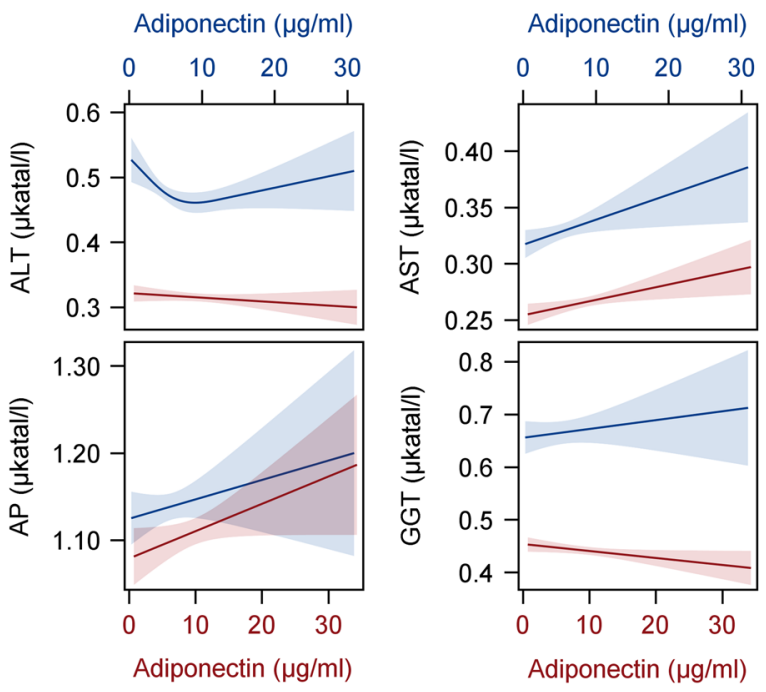

parameters were seen in women. The inverse association between adiponectin and GGT became only apparent in women, but not in men. When analyzing only fasting subjects, the association between adiponectin and AST remained significant in women and changed to a U-shaped association in men. For all other liver enzymes, no significant associations with adiponectin were seen anymore when looking at only fasting subjects (Table 2). Similarly, two further sensitivity analyses revealed very inconsistent results regarding the associations between adiponectin and liver enzymes (data not shown).

In both sexes, a clearly significant inverse association was seen between adiponectin and MRI-quantified liver fat content (Fig. 2 and Table 2). This association remained stable in all observed subpopulations.

Logistic regression analyses confirmed the inverse association between adiponectin and liver fat content. By each increase in serum adiponectin concentrations per $5 \mu \mathrm{g} / \mathrm{mL}$, men and women showed 48 and $42 \%$ lower odds of having hepatic steatosis defined by MRI-quantified liver fat content (Table 3), respectively. Similar results were observed from the ultrasound-based definition of hepatic steatosis (Table 3). Sensitivity analyses with fasting subjects clearly confirmed the results seen in the whole study population (Table 3). Moreover, the presented effect estimates remained stable in the different subpopulations that were examined for sensitivity analyses (data not shown).

\section{Discussion}

In the present study, we used data from a general population to examine the associations of circulating chemerin and adiponectin concentrations with liver enzymes,

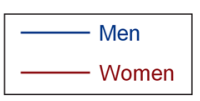

Adiponectin $(\mu \mathrm{g} / \mathrm{ml})$

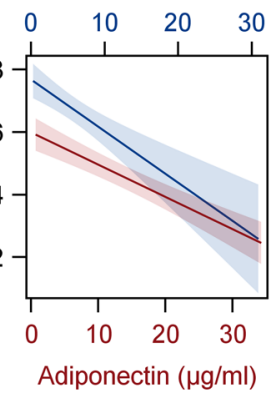

\section{Figure 2}

Associations of serum adiponectin concentrations with liver enzymes and liver fat content (LFC) as calculated by sex-specific quantile regression analyses. Presented is the predicted median together with its corresponding $95 \%$ confidence interval separately for men (blue, $\times 2$ axis) and women (red, $x 1$ axis). Quantile regression models were adjusted for age, waist circumference, glycated hemoglobin, systolic blood pressure, total triglycerides and high-sensitivity C-reactive protein. *Magnetic resonance imaging examinations were performed in a subsample of 840 men and 895 women. ALT, alanine aminotransferase; AP, alkaline phosphatase; AST, aspartate aminotransferase; GGT, gamma glutamyl transferase.
MRI-quantified liver fat content and two different definitions of hepatic steatosis. The analyses regarding the relation of chemerin and adiponectin with liver enzymes showed very divergent findings depending on sex, fasting status and the specific enzyme. More consistent results were seen in the analyses of MRI-quantified liver fat content with strictly inverse associations to adiponectin in both sexes and a positive or U-shaped association to chemerin in men or women, respectively. Likewise, the MRI-based definition of hepatic steatosis revealed the most obvious results: in both sexes high chemerin concentrations were associated with higher odds of hepatic steatosis, whereas high adiponectin concentrations were associated with lower odds of hepatic steatosis. Hence, the two observed adipokines showed opposite effects in relation to hepatic steatosis.

\section{Relation between circulating chemerin concentrations and liver enzymes, MRI-quantified liver fat content and hepatic steatosis}

Most individuals with hepatic steatosis are asymptomatic and hence the liver disease is often accidentally discovered by high concentrations of liver enzymes which are known to indicate a damage of the liver cells $(2,28)$. However, the underlying diseases and conditions that may contribute to high liver enzymes can be manifold (28). Hence, liver enzymes as indicators of liver function are not very specific which may explain the still considerable disagreement on the possible relation between circulating chemerin concentrations and different liver enzymes. Contrary to our results, a broad range of studies have failed to detect significant associations between serum chemerin concentrations and ALT, AST or GGT $(15,29,30)$. 
Beside the potential unspecific nature of liver enzymes, possible reasons for these inconsistent results might be the relatively low number of study participants in previous investigations and/or unconsidered sex effects. To the best of our knowledge, none of the existing studies have performed sex-specific analyses even though evidence exists that CMKLR1 mRNA regulation in chronic liver diseases is influenced by sex (31). Furthermore, our analyses showed that the subjects' fasting status had a strong influence on the observed associations between chemerin concentrations and liver enzymes as the majority of detected associations disappeared when only fasting subjects were considered. In line with this, previous studies which explicitly analyzed only fasting subjects were not able to observe significant associations between serum chemerin concentrations and $\operatorname{ALT}, \operatorname{AST}$ or $\operatorname{GGT}(29,30)$.

Nevertheless, the observed positive association between circulating chemerin concentrations and hepatic steatosis (defined by a MRI-quantified liver fat content $>5 \%$ ) was consistent in both sexes in the present study. Previous studies investigating this association have mainly focused on patients with the clinical diagnosis of NAFLD $(11,12,13,14,15)$ and have detected higher $(11,13,14)$, but also similar $(12,15)$ serum chemerin concentrations in patients with NAFLD compared with healthy controls. Our results were further supported by experimental studies showing elevated hepatic chemerin and CMKLR1 mRNA expression levels in patients with hepatic steatosis compared to healthy controls $(32,33)$ as well as increased expression levels with the severity of hepatic steatosis (32). However, these studies have been contradicted by other investigations which reported that hepatic chemerin and CMKLR1 expressions are reduced in rodent and human fatty liver $(10,34)$. Finally, no correlation between hepatic chemerin mRNA expressions and circulating chemerin concentrations has been detected within previous studies (32). Therefore, changes in chemerin expressions may not correspond to changes in circulating chemerin concentrations. More analyses are needed to clarify the regulation of chemerin expression in the liver and its influence on circulating concentrations.

Besides its potential actions in the liver, chemerin might be involved in the pathogenesis of hepatic steatosis by influencing the innate immune system $(5,35)$. This might happen by its known ability to recruit cells of the innate immune system to sites of tissue injury through activation of CMKLR1 (5). An imbalance of these innate immune cells in the liver is believed to play an important role in the pathogenesis of hepatic steatosis by influencing an excessive production of Th1 cytokines or generating reactive oxygen species (35). Furthermore, circulating chemerin has been observed to be positively correlated with macrophages (7) and a number of pro-inflammatory cytokines (33). In accordance, decreased hepatic mRNA expressions of the pro-inflammatory cytokines TNF $\alpha$ and IL-6 have been detected in CMKLR1-knockout mice compared with WT mice (36). In summary, these studies suggest that the high circulating chemerin concentrations might be explained as an inflammatory response occurring during hepatic steatosis. In the present study, all analyses were adjusted for hsCRP as inflammatory marker. Therefore, we assume that the detected positive association between plasma chemerin concentrations and hepatic steatosis was observed independently of inflammatory processes.

\section{Relation between circulating adiponectin concentrations and liver enzymes, MRI-quantified liver fat content and hepatic steatosis}

Existing human studies dealing with the association between circulating adiponectin and the presence of hepatic steatosis have mainly reported inverse relations $(17,18,20)$ and are therefore in line with our results. Moreover, many investigations have revealed lower circulating adiponectin concentrations together with the grade of hepatic steatosis or the amount of hepatic fat (14, $17,18,19,37)$. However, unlike our results, most of the existing data have shown an inverse association between adiponectin and ALT, AST, AP or GGT $(17,18,20,21)$. Explanations for the mentioned discrepancy between these reports and our results may be found in different adjustment sets applied for multivariable regression analyses and/or unconsidered sex-specific effects. Furthermore, we observed that the associations between circulating adiponectin concentrations and liver enzymes were highly sensitive to the subjects' fasting status, an aspect which is often not considered $(18,21)$ and which hamper the comparison between studies. Those few studies that explicitly analyzed only fasting subjects revealed a significant inverse association of adiponectin with ALT or GGT $(17,20)$. Although we observed nonsignificant associations between adiponectin and those liver enzymes, a strong and significant inverse association between adiponectin concentrations and MRI-quantified liver fat content was seen (independently from subjects' fasting status).

In line with our results, recent experimental studies have shown that adiponectin as well as its two main receptors, AdipoR1 and AdipoR2, were expressed by

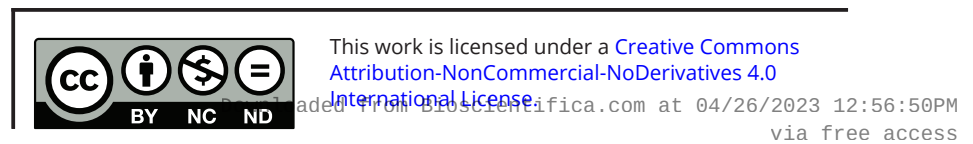


different liver cells (16) and that the expression levels were inversely associated with the severity of hepatic steatosis (38). Thus, adiponectin seems to have hepatoprotective actions (16), but as described in several in vitro and in vivo studies, the potential mechanisms through which adiponectin may exert these protective properties can be manifold.

Similar to chemerin, adiponectin appears to play an important role during inflammatory processes. In this regard, it was observed that adiponectin inhibits the expression and actions of pro-inflammatory cytokines (39), while inducing the production of anti-inflammatory cytokines (37). As pro-inflammatory cytokines are known to be key mediators of liver diseases (40), the antiinflammatory mechanisms of adiponectin might explain the observed inverse association between its circulating concentrations and hepatic steatosis. However, all our analyses were adjusted for hsCRP and thus it is likely that the detected inverse association between adiponectin and hepatic steatosis was observed independently of inflammatory mechanisms.

Other experimental studies suggest that the hepatoprotective actions of adiponectin might be explained by its involvement in the process of fatty acid synthesis (41), its influence on proliferation and migration of hepatic stellate cells (42) or its ability to inhibit apoptotic cell death of hepatocytes (43). Taken together, adiponectin seems to be able to control a number of different mechanisms in the liver, but further experimental studies are urgently needed to clarify the exact underlying mechanisms.

\section{Definition of hepatic steatosis}

In order to validate our findings, we used two different ways to define hepatic steatosis. In a first setting, we defined hepatic steatosis by the presence of an ultrasonographically assessed hyperechogenic liver. The second definition of hepatic steatosis was based on MRI examinations. With the exception of the association between chemerin and hepatic steatosis in women, all analyses revealed clearly comparable results for both definitions of hepatic steatosis. Only the observed effect estimates were slightly higher when using the MRI-based definition compared to the ultrasound-based definition of hepatic steatosis. However, in women, it was not possible to validate the observed positive association between chemerin and the MRI-based definition of hepatic steatosis when using the ultrasound-based definition, for reasons currently unknown. In general, previous studies have reported that MRI methods are highly accurate and reproducible in measuring liver fat content $(44,45)$. Therefore, we assume that the detected association between chemerin and MRI-based definition of hepatic steatosis is reliable.

\section{Strengths and limitations}

The key strengths of the present study are the large sample size with a total of 3872 subjects and the consistency and high quality with which the data was collected. In order to verify our results, we measured different liver enzymes and used two imaging methods to estimate hepatic steatosis. Moreover, we investigated the associations of circulating chemerin and adiponectin with MRIquantified liver fat content as well as possible sex-specific differences in the associations of circulating chemerin concentrations with hepatic steatosis for the first time. Limitations arise from the cross-sectional study design, which prevents the assessment of causal relations and does not allow announcing the mechanisms behind the observed associations. Furthermore, it was not possible to examine any potential influence of the fasting status at the time of the MRI examinations on our results as this information was missing.

\section{Conclusion}

In conclusion, our study is the first that reports significant associations of circulating chemerin and adiponectin concentrations with MRI-quantified liver fat content in both sexes using data from a large population-based study. Interestingly, chemerin and adiponectin exert opposite effects in this context. The existing literature suggests that both adipokines are involved in the inflammatory response that develops during hepatic steatosis (14). However, in the present study, the associations between these adipokines and hepatic steatosis were observed independently of inflammatory disorders. This suggests that the relation between the analyzed adipokines and hepatic steatosis cannot be explained by inflammatory changes alone. Future longitudinal examinations evaluating the predictive and therapeutic meaning of adiponectin and chemerin in hepatic steatosis are urgently needed. Furthermore, experimental studies are necessary to clarify the complex paracrine and endocrine mechanisms underlying the link between circulating adipokine concentrations and hepatic steatosis.
This work is licensed under a Creative Commons Attribution-NonCommercial-NoDerivatives 4.0 elnternationad ticense ifica.com at 04/26/2023 12:56:50PM 


\section{Declaration of interest}

The authors declare that there is no conflict of interest that could be perceived as prejudicing the impartiality of the research reported.

\section{Funding}

This work was funded by grants from the German Federal Ministry of Education and Research (BMBF, Grants 01ZZ0403, 01ZZ0103, 01Gl0883), the Ministry for Education, Research and Cultural Affairs as well as the Ministry of Social Affairs of the Federal State of Mecklenburg-West Pomerania.

\section{References}

1 Kojima S, Watanabe N, Numata M, Ogawa T \& Matsuzaki S. Increase in the prevalence of fatty liver in Japan over the past 12 years: analysis of clinical background. Journal of Gastroenterology 200338 954-961. (https://doi.org/10.1007/s00535-003-1178-8)

2 Angulo P. Nonalcoholic fatty liver disease. New England Journal of Medicine 2002346 1221-1231. (https://doi.org/10.1056/ NEJMra011775)

3 Jarrar MH, Baranova A, Collantes R, Ranard B, Stepanova M, Bennett C, Fang Y, Elariny H, Goodman Z, Chandhoke V, et al. Adipokines and cytokines in non-alcoholic fatty liver disease. Alimentary Pharmacology and Therapeutics 200827 412-421. (https:// doi.org/10.1111/j.1365-2036.2007.03586.x)

4 Marra F \& Bertolani C. Adipokines in liver diseases. Hepatology 2009 50 957-969. (https://doi.org/10.1002/hep.23046)

5 Rourke JL, Dranse HJ \& Sinal CJ. Towards an integrative approach to understanding the role of chemerin in human health and disease. Obesity Reviews 201314 245-262. (https://doi.org/10.1111/ obr.12009)

6 Bozaoglu K, Bolton K, McMillan J, Zimmet P, Jowett J, Collier G, Walder K \& Segal D. Chemerin is a novel adipokine associated with obesity and metabolic syndrome. Endocrinology 2007148 4687-4694. (https://doi.org/10.1210/en.2007-0175)

7 Sell H, Divoux A, Poitou C, Basdevant A, Bouillot JL, Bedossa P, Tordjman J, Eckel J \& Clement K. Chemerin correlates with markers for fatty liver in morbidly obese patients and strongly decreases after weight loss induced by bariatric surgery. Journal of Clinical Endocrinology and Metabolism 201095 2892-2896. (https://doi. org/10.1210/jc.2009-2374)

8 Skuja I, Stukena I \& Lejnieks A. Chemerin relationship with glucose and lipid metabolism in clinically asymptomatic patients. Family Medicine and Medical Science Research 20155 1-6. (https://doi. org/10.4172/2327-4972.1000193)

9 Zylla S, Pietzner M, Kühn JP, Völzke H, Dörr M, Nauck M \& Friedrich N. Serum chemerin is associated with inflammatory and metabolic parameters-results of a population-based study. Obesity 201725 468-475. (https://doi.org/10.1002/oby.21735)

10 Wanninger J, Bauer S, Eisinger K, Weiss TS, Walter R, Hellerbrand C, Schäffler A, Higuchi A, Walsh K \& Buechler C. Adiponectin upregulates hepatocyte CMKLR1 which is reduced in human fatty liver. Molecular and Cellular Endocrinology 2012349 248-254. (https:// doi.org/10.1016/j.mce.2011.10.032)

11 Yilmaz Y, Yonal O, Kurt R, Alahdab YO, Eren F, Ozdogan O, Celikel CA, Imeryuz N, Kalayci C \& Avsar E. Serum levels of omentin, chemerin and adipsin in patients with biopsy-proven nonalcoholic fatty liver disease. Scandinavian Journal of Gastroenterology 201146 91-97. (https://doi.org/10.3109/00365521.2010.516452)

12 Ye Z, Wang S, Yang Z, He M, Zhang S, Zhang W, Wen J, Li Q, Huang $Y$, Wang $X$, et al. Serum lipocalin-2, cathepsin $S$ and chemerin levels and nonalcoholic fatty liver disease. Molecular Biology Reports 201441 1317-1323. (https://doi.org/10.1007/s11033-013-2977-5)
13 Kukla M, Zwirska-Korczala K, Hartleb M, Waluga M, Chwist A, Kajor M, Ciupinska-Kajor M, Berdowska A, Wozniak-Grygiel E \& Buldak R. Serum chemerin and vaspin in non-alcoholic fatty liver disease. Scandinavian Journal of Gastroenterology 201045 235-242. (https://doi.org/10.3109/00365520903443852)

14 Bekaert M, Ouwens DM, Horbelt T, Van de Velde F, Fahlbusch P, Herzfeld de Wiza D, Van Nieuwenhove Y, Calders P, Praet M, Hoorens A, et al. Reduced expression of chemerin in visceral adipose tissue associates with hepatic steatosis in patients with obesity. Obesity 201624 2544-2552. (https://doi.org/10.1002/oby.21674)

15 Pohl R, Haberl EM, Rein-Fischboeck L, Zimny S, Neumann M, Aslanidis C, Schacherer D, Krautbauer S, Eisinger K, Weiss TS, et al. Hepatic chemerin mRNA expression is reduced in human nonalcoholic steatohepatitis. European Journal of Clinical Investigation 201747 7-18. (https://doi.org/10.1111/eci.12695)

16 Polyzos SA, Kountouras J, Zavos C \& Tsiaousi E. The role of adiponectin in the pathogenesis and treatment of non-alcoholic fatty liver disease. Diabetes, Obesity and Metabolism 201012 365-383. (https://doi.org/10.1111/j.1463-1326.2009.01176.x)

17 Bugianesi E, Pagotto U, Manini R, Vanni E, Gastaldelli A, de Iasio R, Gentilcore E, Natale S, Cassader M, Rizzetto M, et al. Plasma adiponectin in nonalcoholic fatty liver is related to hepatic insulin resistance and hepatic fat content, not to liver disease severity. Journal of Clinical Endocrinology and Metabolism 200590 3498-3504. (https://doi.org/10.1210/jc.2004-2240)

18 Flechtner-Mors M, George SN, Oeztuerk S, Haenle MM, Koenig W, Imhof A, Boehm BO, Graeter T, Mason RA, Kratzer W, et al. Association of adiponectin with hepatic steatosis: a study of 1,349 subjects in a random population sample. BMC Research Notes 20147 1-8. (https://doi.org/10.1186/1756-0500-7-207)

19 Bajaj M, Suraamornkul S, Piper P, Hardies LJ, Glass L, Cersosimo E, Pratipanawatr T, Miyazaki Y \& DeFronzo RA. Decreased plasma adiponectin concentrations are closely related to hepatic fat content and hepatic insulin resistance in pioglitazone-treated type 2 diabetic patients. Journal of Clinical Endocrinology and Metabolism 200489 200-206. (https://doi.org/10.1210/jc.2003-031315)

20 Targher G, Bertolini L, Scala L, Poli F, Zenari L \& Falezza G. Decreased plasma adiponectin concentrations are closely associated with nonalcoholic hepatic steatosis in obese individuals. Clinical Endocrinology 200461 700-703. (https://doi.org/10.1111/j.13652265.2004.02151.x)

21 Lopez-Bermejo A, Botas P, Funahashi T, Delgado E, Kihara S, Ricart W \& Fernandez-Real JM. Adiponectin, hepatocellular dysfunction and insulin sensitivity. Clinical Endocrinology 200460 256-263. (https:// doi.org/10.1046/j.1365-2265.2004.01977.x)

22 Völzke H, Alte D, Schmidt CO, Radke D, Lorbeer R, Friedrich N, Aumann N, Lau K, Piontek M, Born G, et al. Cohort profile: the study of health in Pomerania. International Journal of Epidemiology 201140 294-307. (https://doi.org/10.1093/ije/dyp394)

23 Völzke H, Nauck M, Rettig R, Dörr M, Higham C, Brabant G \& Wallaschofski H. Association between hepatic steatosis and serum IGF1 and IGFBP-3 levels in a population-based sample. European Journal of Endocrinology 2009161 705-713. (https://doi.org/10.1530/ EJE-09-0374)

24 Kühn JP, Hernando D, Mensel B, Krüger PC, Ittermann T, Mayerle J, Hosten N \& Reeder SB. Quantitative chemical shift-encoded MRI is an accurate method to quantify hepatic steatosis. Journal of Magnetic Resonance Imaging 201439 1494-1501. (https://doi.org/10.1002/ jmri.24289)

25 Reeder SB \& Sirlin CB. Quantification of liver fat with magnetic resonance imaging. Magnetic Resonance Imaging Clinics of North America 201018 337-357. (https://doi.org/10.1016/j. mric.2010.08.013)

26 Kühn JP, Hernando D, Munoz del Rio A, Evert M, Kannengiesser S, Völzke H, Mensel B, Puls R, Hosten N \& Reeder SB. Effect of multipeak spectral modeling of fat for liver iron and fat 
quantification: correlation of biopsy with MR imaging results. Radiology 2012265 133-142. (https://doi.org/10.1148/ radiol.12112520)

27 Tan BK, Chen J, Farhatullah S, Adya R, Kaur J, Heutling D, Lewandowski KC, O'Hare JP, Lehnert H \& Randeva HS. Insulin and metformin regulate circulating and adipose tissue chemerin. Diabetes 200958 1971-1977. (https://doi.org/10.2337/db08-1528)

28 Hall P \& Cash J. What is the real function of the liver 'function' tests? Ulster Medical Journal 201281 30-36.

29 Kim SH, Lee SH, Ahn KY, Lee DH, Suh YJ, Cho SG, Choi YJ, Lee DH, Lee SY, Hong SB, et al. Effect of lifestyle modification on serum chemerin concentration and its association with insulin sensitivity in overweight and obese adults with type 2 diabetes. Clinical Endocrinology 201480 825-833. (https://doi.org/10.1111/cen.12249)

30 Hatziagelaki E, Herder C, Tsiavou A, Teichert T, Chounta A, Nowotny P, Pacini G, Dimitriadis G \& Roden M. Serum chemerin concentrations associate with beta-cell function, but not with insulin resistance in individuals with non-alcoholic fatty liver disease (NAFLD). PLOS ONE 201510 1-11. (https://doi.org/10.1371/journal. pone.0124935)

31 Neumann M, Meier EM, Rein-Fischboeck L, Krautbauer S, Eisinger K, Aslanidis C, Pohl R, Weiss TS \& Buechler C. Chemokine-like receptor 1 mRNA weakly correlates with non-alcoholic steatohepatitis score in male but not female individuals. International Journal of Molecular Sciences 201617 1-10. (https://doi.org/10.3390/ijms17081335)

32 Döcke S, Lock JF, Birkenfeld AL, Hoppe S, Lieske S, Rieger A, Raschzok N, Sauer IM, Florian S, Osterhoff MA, et al. Elevated hepatic chemerin mRNA expression in human non-alcoholic fatty liver disease. European Journal of Endocrinology 2013169 547-557. (https:// doi.org/10.1530/EJE-13-0112)

33 Krautbauer S, Wanninger J, Eisinger K, Hader Y, Beck M, Kopp A, Schmid A, Weiss TS, Dorn C \& Buechler C. Chemerin is highly expressed in hepatocytes and is induced in non-alcoholic steatohepatitis liver. Experimental and Molecular Pathology 201395 199-205. (https://doi.org/10.1016/j.yexmp.2013.07.009)

34 Deng Y, Wang H, Lu Y, Liu S, Zhang Q, Huang J, Zhu R, Yang J, Zhang R, Zhang D, et al. Identification of chemerin as a novel FXR target gene down-regulated in the progression of nonalcoholic steatohepatitis. Endocrinology 2013154 1794-1801. (https://doi. org/10.1210/en.2012-2126)

35 Zhan YT \& An W. Roles of liver innate immune cells in nonalcoholic fatty liver disease. World Journal of Gastroenterology 201016 46524660. (https://doi.org/10.3748/wjg.v16.i37.4652)

36 Ernst MC, Haidl ID, Zuniga LA, Dranse HJ, Rourke JL, Zabel BA, Butcher EC \& Sinal CJ. Disruption of the chemokine-like receptor-1
(CMKLR1) gene is associated with reduced adiposity and glucose intolerance. Endocrinology 2012153 672-682. (https://doi. org/10.1210/en.2011-1490)

37 Mandal P, Park PH, McMullen MR, Pratt BT \& Nagy LE. The antiinflammatory effects of adiponectin are mediated via a heme oxygenase-1-dependent pathway in rat Kupffer cells. Hepatology 2010 51 1420-1429. (https://doi.org/10.1002/hep.23427)

38 Kaser S, Moschen A, Cayon A, Kaser A, Crespo J, Pons-Romero F, Ebenbichler CF, Patsch JR \& Tilg H. Adiponectin and its receptors in non-alcoholic steatohepatitis. Gut 200554 117-121. (https://doi. org/10.1136/gut.2003.037010)

39 Masaki T, Chiba S, Tatsukawa H, Yasuda T, Noguchi H, Seike M \& Yoshimatsu H. Adiponectin protects LPS-induced liver injury through modulation of TNF-alpha in KK-Ay obese mice. Hepatology 200440 177-184. (https://doi.org/10.1002/hep.20282)

40 Carter-Kent C, Zein NN \& Feldstein AE. Cytokines in the pathogenesis of fatty liver and disease progression to steatohepatitis: implications for treatment. American Journal of Gastroenterology 2008 103 1036-1042. (https://doi.org/10.1111/j.1572-0241.2007.01709.x)

41 Awazawa M, Ueki K, Inabe K, Yamauchi T, Kaneko K, Okazaki Y, Bardeesy N, Ohnishi S, Nagai R \& Kadowaki T. Adiponectin suppresses hepatic SREBP1c expression in an AdipoR1/LKB1/ AMPK dependent pathway. Biochemical and Biophysical Research Communications 2009382 51-56. (https://doi.org/10.1016/j. bbrc.2009.02.131)

42 Kamada Y, Tamura S, Kiso S, Matsumoto H, Saji Y, Yoshida Y, Fukui K, Maeda N, Nishizawa H, Nagaretani H, et al. Enhanced carbon tetrachloride-induced liver fibrosis in mice lacking adiponectin. Gastroenterology 2003125 1796-1807. (https://doi.org/10.1053/j. gastro.2003.08.029)

43 Jung TW, Lee YJ, Lee MW, Kim SM \& Jung TW. Full-length adiponectin protects hepatocytes from palmitate-induced apoptosis via inhibition of c-Jun NH2 terminal kinase. FEBS Journal 2009276 2278-2284. (https://doi.org/10.1111/j.17424658.2009.06955.x)

44 Lee SS, Park SH, Kim HJ, Kim SY, Kim MY, Kim DY, Suh DJ, Kim KM, Bae MH, Lee JY, et al. Non-invasive assessment of hepatic steatosis: prospective comparison of the accuracy of imaging examinations. Journal of Hepatology 201052 579-585. (https://doi.org/10.1016/j. jhep.2010.01.008)

45 van Werven JR, Marsman HA, Nederveen AJ, Smits NJ, ten Kate FJ, van Gulik TM \& Stoker J. Assessment of hepatic steatosis in patients undergoing liver resection: comparison of US, CT, T1-weighted dualecho MR imaging, and point-resolved 1H MR spectroscopy. Radiology 2010256 159-168. (https://doi.org/10.1148/radiol.10091790)

Received in final form 17 June 2019

Accepted 2 July 2019

Accepted Preprint published online 2 July 2019 https://ec.bioscientifica.com https://doi.org/10.1530/EC-19-0300
(C) 2019 The authors Published by Bioscientifica Ltd
This work is licensed under a Creative Commons Attribution-NonCommercial-NoDerivatives 4.0 elnternationad ticense ifica . com at 04/26/2023 12:56:50PM 